PROCEEDINGS OF THE

AMERICAN MATHEMATICAL SOCIETY

Volume 129, Number 3, Pages 855-859

S 0002-9939(00)05650-1

Article electronically published on September 20, 2000

\title{
INEQUALITIES OF REID TYPE AND FURUTA
}

\author{
C.-S. LIN \\ (Communicated by Jonathan M. Borwein) \\ Dedicated to Professor Jone Lin on his retirement
}

\begin{abstract}
Two of the most useful inequality formulas for bounded linear operators on a Hilbert space are the Löwner-Heinz and Reid's inequalities. The first inequality was generalized by Furuta (so called the Furuta inequality in the literature). We shall generalize the second one and obtain its related results. It is shown that these two generalized fundamental inequalities are all equivalent to one another.
\end{abstract}

\section{NotATions AND InTRODUCTION}

Throughout the paper we use capital letters to denote bounded linear operators acting on a Hilbert space H. $T$ is positive (written $T \geq O$ ) in case $(T x, x) \geq 0$ for all $x \in \mathrm{H}$. If $S$ and $T$ are Hermitian, we write $T \geq S$ in case $T-S \geq O . T=U|T|$ is the polar decomposition of $T$ with $U$ the partial isometry, whereas $|T|$ is the positive square root of the operator $T^{*} T$, and $U^{*} U$ is the initial projection. Let $I$ denote the identity operator. Under the polar decomposition of $T$ we recall a well-known relation: $\left|T^{*}\right|^{q}=U|T|^{q} U^{*}$ for $q>0$ [1, p. 752].

The aim of this article is to define and characterize a generalized Reid inequality. Consequently, related inequalities and improved inequalities are given. Recall that if $S \geq O$ and $S K$ is Hermitian, then for $x \in \mathrm{H},|(S K x, x)| \leq\|K\|(S x, x)$ is Reid's inequality [8], and the inequality was sharpened by Halmos [4, pp. 51, 244], where $\|K\|$ is replaced by the spectral radius of $K$. Let $\alpha, \beta \in[0,1]$ with $\alpha+\beta \geq 1$. For $x, y \in \mathrm{H}$ let us call $\left|\left(S K|S K|^{\alpha+\beta-1} x, y\right)\right| \leq\|K\|^{\alpha+\beta}\left\|S^{\alpha} x\right\|\left\|S^{\beta} y\right\|$ an extended Reid's inequality. We shall call, for an obvious reason, the inequality (2) in Theorem 1 below a generalized Reid inequality, whereas (1) is the well-known altered Furuta inequality [2. Our main result is characterizations of this inequality.

\section{MAIN THEOREM}

Theorem 1. Let $S \geq O, S K$ be Hermitian, and let $S K=V|S K|$ be the polar decomposition. Then the following inequalities hold and follow from each other for

Received by the editors May 25, 1999.

1991 Mathematics Subject Classification. Primary 47A63.

Key words and phrases. Positive operator, Hermitian operator, polar decomposition, LöwnerHeinz inequality, Reid's inequality, Furuta's inequality, contraction.

(c)2000 American Mathematical Society 
all $x, y \in H, r, s \geq 0, p, q \geq 1$, and $\alpha, \beta \in[0,1]$ with $(1+2 r) \alpha+(1+2 s) \beta \geq 1$.

(1) $\left(B^{r} A^{p} B^{r}\right)^{(1+2 r) \alpha /(p+2 r)} \geq B^{(1+2 r) \alpha}$ if $A \geq B \geq O$;

(2) $\left|\left(S K|S K|^{(1+2 r) \alpha+(1+2 s) \beta-1} x, y\right)\right|^{2}$

$\leq\|K\|^{a}\left(\left(|S K|^{2 r} S^{2 p}|S K|^{2 r}\right)^{(1+2 r) \alpha /(p+2 r)} x, x\right)$

$\cdot\left(\left(|S K|^{2 s} S^{2 q}|S K|^{2 s}\right)^{(1+2 s) \beta /(q+2 s)} y, y\right) ;$

(3) $\left|\left(S K|S K|^{(1+2 r) \alpha+(1+2 s) \beta-1} x, y\right)\right|^{2}$

$\leq\|K\|^{b}\left(\left(|S K|^{2 r} S^{2 p}|S K|^{2 r}\right)^{(1+2 r) \alpha /(p+2 r)} x, x\right)\left(|S K|^{2(1+2 s) \beta} y, y\right) ;$

(4) $\left|\left(S K|S K|^{(1+2 r) \alpha+(1+2 s) \beta-1} x, y\right)\right|^{2}$

$\leq\|K\|^{c}\left(|S K|^{2(1+2 r) \alpha} x, x\right)\left(\left(|S K|^{2 s} S^{2 q}|S K|^{2 s}\right)^{(1+2 s) \beta /(q+2 s)} y, y\right) ;$

(5) $\left|\left(|S K|^{(1+2 r) \alpha+(1+2 s) \beta} x, y\right)\right|^{2}$

$\leq\|K\|^{a}\left(\left(|S K|^{2 r} S^{2 p}|S K|^{2 r}\right)^{(1+2 r) \alpha /(p+2 r)} x, x\right)$

$\cdot\left(\left(|S K|^{2 s} S^{2 q}|S K|^{2 s}\right)^{(1+2 s) \beta /(q+2 s)} y, y\right)$;

(6) $\left|\left(|S K|^{(1+2 r) \alpha+(1+2 s) \beta} x, y\right)\right|^{2}$

$\leq\|K\|^{b}\left(\left(|S K|^{2 r} S^{2 p}|S K|^{2 r}\right)^{(1+2 r) \alpha /(p+2 r)} x, x\right)\left(|S K|^{2(1+2 s) \beta} y, y\right) ;$

(7) $\left|\left(|S K|^{(1+2 r) \alpha+(1+2 s) \beta} x, y\right)\right|^{2}$

$\leq\|K\|^{c}\left(|S K|^{2(1+2 r) \alpha} x, x\right)\left(\left(|S K|^{2 s} S^{2 q}|S K|^{2 s}\right)^{(1+2 s) \beta /(q+2 s)} y, y\right)$,

where $b=\frac{2 p(1+2 r) \alpha}{p+2 r}, c=\frac{2 q(1+2 s) \beta}{q+2 s}$, and $a=b+c=\frac{2 p(1+2 r) \alpha}{p+2 r}+\frac{2 q(1+2 s) \beta}{q+2 s}$.

Proof. (1) implies (2). As $K /\|K\|$ is a contraction, i.e., $K K^{*} \leq\|K\|^{2}$, we have

$$
O \leq \frac{|S K|^{2}}{\|K\|^{2}}=\frac{\left|(S K)^{*}\right|^{2}}{\|K\|^{2}}=\frac{S K K^{*} S}{\|K\|^{2}} \leq S^{2}
$$

Thus, inequality (1) implies that

$$
|S K|^{2(1+2 r) \alpha} \leq\|K\|^{b}\left(|S K|^{2 r} S^{2 p}|S K|^{2 r}\right)^{(1+2 r) \alpha /(p+2 r)},
$$

from which we have (replacing $\alpha$ by $\beta, p$ by $q$, and $r$ by $s$ )

$$
|S K|^{2(1+2 s) \beta} \leq\|K\|^{c}\left(|S K|^{2 s} S^{2 q}|S K|^{2 s}\right)^{(1+2 s) \beta /(q+2 s)},
$$

where $b=\frac{2 p(1+2 r) \alpha}{p+2 r}$ and $c=\frac{2 q(1+2 s) \beta}{q+2 s}$. To prove the inequality (2) we proceed as follows. Since

$$
\begin{aligned}
S K & |S K|^{(1+2 r) \alpha+(1+2 s) \beta-1}=V|S K|^{(1+2 r) \alpha+(1+2 s) \beta} \\
& =V|S K|^{(1+2 s) \beta} V^{*} V|S K|^{(1+2 r) \alpha}=|S K|^{(1+2 s) \beta} V|S K|^{(1+2 r) \alpha},
\end{aligned}
$$


it follows that

$$
\begin{aligned}
\mid(S K & \left.|S K|^{(1+2 r) \alpha+(1+2 s) \beta-1} x, y\right)\left.\right|^{2} \\
& =\left|\left(|S K|^{(1+2 s) \beta} V|S K|^{(1+2 r) \alpha} x, y\right)\right|^{2} \\
& \leq\left\|V|S K|^{(1+2 r) \alpha} x\right\|^{2}\left\||S K|^{(1+2 s) \beta} y\right\|^{2} \\
& =\left(|S K|^{2(1+2 r) \alpha} x, x\right)\left(|S K|^{2(1+2 s) \beta} y, y\right) \\
& \leq\|K\|^{a}\left(\left(|S K|^{2 r} S^{2 p}|S K|^{2 r}\right)^{(1+2 r) \alpha /(p+2 r)} x, x\right) \\
& \cdot\left(\left(|S K|^{2 s} S^{2 q}|S K|^{2 s}\right)^{(1+2 s) \beta /(q+2 s)} y, y\right)
\end{aligned}
$$

with $a=b+c=\frac{2 p(1+2 r) \alpha}{p+2 r}+\frac{2 q(1+2 s) \beta}{q+2 s}$.

(1) implies (3) and (4). Each implication is easily seen from the proof above.

(2) implies (1). We may assume without loss of generality that $A$ is invertible. Since $A \geq B \geq O, A^{-1 / 2} B^{1 / 2} B^{1 / 2} A^{-1 / 2} \leq I$. Let $K=A^{-1 / 2} B^{1 / 2}$, which is obviously a contraction. Also let $S=A^{1 / 2} \geq O$. Then $S K=B^{1 / 2} \geq O$. Therefore, if we use these substitutions and let $s=r, \beta=\alpha, q=p$, and $y=x$ in (2), then

$$
\left|\left(B^{(1+2 r) \alpha} x, x\right)\right|^{2} \leq\left(\left(B^{r} A^{p} B^{r}\right)^{(1+2 r) \alpha /(p+2 r)} x, x\right)^{2}
$$

as $\|K\|^{a} \leq 1$, which implies Furuta's inequality.

(3) implies (1). By the same substitutions as in the proof "(2) implies (1)" (3) becomes

$$
\left|\left(B^{(1+2 r) \alpha} x, x\right)\right|^{2} \leq\left(\left(B^{2 r} A^{2 p} B^{2 r}\right)^{(1+2 r) \alpha /(p+2 r)} x, x\right)\left(B^{(1+2 r) \alpha} x, x\right),
$$

and (1) follows.

(4) implies (1). The proof is similar to "(3) implies (1)".

Now, since

$$
\left|\left(|S K|^{(1+2 r) \alpha+(1+2 s) \beta} x, y\right)\right|^{2} \leq\left(|S K|^{2(1+2 r) \alpha} x, x\right)\left(|S K|^{2(1+2 s) \beta} y, y\right),
$$

statements (1), (5), (6) and (7) are all equivalent by repeating the argument in the proof above, and this completes the proof of Theorem 1.

\section{Consequences of Theorem 1}

Let $s=r=0$ in Theorem 1. Then we obtain characterizations of an extended Reid's inequality (2) in Corollary 1 below, whereas (1) is called the Löwner-Heinz inequality in the literature.

Corollary 1. Let $S \geq O, S K$ be Hermitian, and let $S K=V|S K|$ be the polar decomposition. Then the following inequalities hold and follow from each other for all $x, y \in H$, and $\alpha, \beta \in[0,1]$ with $\alpha+\beta \geq 1$.

(1) $A^{\alpha} \geq B^{\alpha}$ if $A \geq B \geq O$;

(2) $\left|\left(S \bar{K}|S K|^{\alpha+\beta}-1 \bar{x}, y\right)\right| \leq\|K\|^{\alpha+\beta}\left\|S^{\alpha} x\right\|\left\|S^{\beta} y\right\|$;

(3) $\left|\left(S K|S K|^{\alpha+\beta-1} x, y\right)\right| \leq\|K\|^{\alpha}\left\|S^{\alpha} x\right\|\left\||S K|^{\beta} y\right\|$;

(4) $\left|\left(S K|S K|^{\alpha+\beta-1} x, y\right)\right| \leq\|K\|^{\beta}\left\||S K|^{\alpha} x\right\|\left\|S^{\beta} y\right\|$;

(5) $\left|\left(|S K|^{\alpha+\beta} x, y\right)\right| \leq\|K\|^{\alpha+\beta}\left\|S^{\alpha} x\right\|\left\|S^{\beta} y\right\|$;

(6) | (| SK $\left.\left.\right|^{\alpha+\beta} x, y\right)\left|\leq\|K\|^{\alpha}\left\|S^{\alpha} x\right\|\left\||S K|^{\beta} y\right\|\right.$;

(7) $\left|\left(|S K|^{\alpha+\beta} x, y\right)\right| \leq\|K\|^{\beta}\left\||S K|^{\alpha} x\right\|\left\|S^{\beta} y\right\|$. 
Let $\beta=1-\alpha$ in Corollary 1 ; then we have another set of seven inequalities equivalent to one another, and we shall omit the details. Consequently, we have several characterizations of Reid's inequality (2) in Corollary 2 below. Note that inequality (1) was first proved by Löwner [7].

Corollary 2. Let $S \geq O, S K$ be Hermitian, and let $S K=V|S K|$ be the polar decomposition. Then the following inequalities hold and follow from each other for all $x \in H$.

(1) $A^{1 / 2} \geq B^{1 / 2}$ if $A \geq B \geq O$;

(2) $|(S K x, x)| \leq\|K\|(S x, x)$;

(3) $|(S K x, x)| \leq\|K\|^{1 / 2}(|S K| x, x)^{1 / 2}(S x, x)^{1 / 2}$;

(4) $|(|S K| x, x)| \leq\|K\|(S x, x)$;

(5) $|(|S K| x, x)| \leq\|K\|^{1 / 2}(|S K| x, x)^{1 / 2}(S x, x)^{1 / 2}$.

Note that if we put $\alpha=\frac{1}{2}$ in Corollary 1 , then inequality (1) in Corollary 2 is also equivalent to many other inequalities. In fact, there are uncountably many inequalities as long as $\frac{1}{2}+\beta \geq 1$ for $\beta \in[0,1]$. Thus, it is interesting to know that Reid's inequality (2) in Corollary 2 is equivalent to uncountably many inequalities.

\section{IMPROVEMENT OF REID TYPE INEQUALITIES}

The proof of the next result is included in that of Theorem 1. It is an improvement of a generalized Reid's inequality, and we shall omit the proof.

Theorem 2. Let $S \geq O, S K$ be Hermitian, and let $S K=V|S K|$ be the polar decomposition. Then the following inequalities hold for all $x, y \in H, r, s \geq 0$, $p, q \geq 1$, and $\alpha, \beta \in[0,1]$ with $(1+2 r) \alpha+(1+2 s) \beta \geq 1$ :

$$
\begin{aligned}
\left|\left(S K|S K|^{(1+2 r) \alpha+(1+2 s) \beta-1} x, y\right)\right|^{2} \leq\left(|S K|^{2(1+2 r) \alpha} x, x\right)\left(|S K|^{2(1+2 s) \beta} y, y\right) \\
\leq\left\{\begin{array}{l}
\|K\|^{b}\left(\left(|S K|^{2 r} S^{2 p}|S K|^{2 r}\right)^{(1+2 r) \alpha /(p+2 r)} x, x\right)\left(|S K|^{2(1+2 s) \beta} y, y\right) \\
\|K\|^{c}\left(|S K|^{2(1+2 r) \alpha} x, x\right)\left(\left(|S K|^{2 s} S^{2 q}|S K|^{2 s}\right)^{(1+2 s) \beta /(q+2 s)} y, y\right)
\end{array}\right\} \\
\leq\|K\|^{a}\left(\left(|S K|^{2 r} S^{2 p}|S K|^{2 r}\right)^{(1+2 r) \alpha /(p+2 r)} x, x\right) \\
\cdot\left(\left(|S K|^{2 s} S^{2 q}|S K|^{2 s}\right)^{(1+2 s) \beta /(q+2 s)} y, y\right) .
\end{aligned}
$$

where $b=\frac{2 p(1+2 r) \alpha}{p+2 r}, c=\frac{2 q(1+2 s) \beta}{q+2 s}$, and $a=\frac{2 p(1+2 r) \alpha}{p+2 r}+\frac{2 q(1+2 s) \beta}{q+2 s}$.

Also, the first term $\left|\left(S K|S K|^{(1+2 r) \alpha+(1+2 s) \beta-1} x, y\right)\right|^{2}$ above may be replaced by the term $\left|\left(|S K|^{(1+2 r) \alpha+(1+2 s) \beta} x, y\right)\right|^{2}$ without changing relations.

Consequently, we have the next improved extended Reid's inequality and Reid's inequality.

Corollary 3. Let $S \geq O, S K$ be Hermitian, and let $S K=V|S K|$ be the polar decomposition. Then the following inequalities hold for all $x, y \in H$, and $\alpha, \beta \in[0,1]$ with $\alpha+\beta \geq 1$.

$$
\begin{aligned}
(1) & \left|\left(S K|S K|^{\alpha+\beta-1} x, y\right)\right| \leq\left\||S K|^{\alpha} x\right\|\left\||S K|^{\beta} y\right\| \\
& \leq\left\{\begin{array}{l}
\|K\|^{\alpha}\left\|S^{\alpha} x\right\|\left\||S K|^{\beta} y\right\| \\
\|K\|^{\beta}\left\||S K|^{\alpha} x\right\|\left\|S^{\beta} y\right\|
\end{array}\right\} \\
& \leq\|K\|^{\alpha+\beta}\left\|S^{\alpha} x\right\|\left\|S^{\beta} y\right\| .
\end{aligned}
$$


Also, the first term $\left|\left(S K|S K|^{\alpha+\beta-1} x, y\right)\right|$ above may be replaced by the term | $\left(|S K|^{\alpha+\beta} x, y\right) \mid$ without changing relations.

$$
\begin{aligned}
(2) \mid & (S K x, x) \mid \leq(|S K| x, x) \\
& \leq\|K\|^{1 / 2}\left\||S K|^{1 / 2} x\right\|\left\|S^{1 / 2} x\right\| \leq\|K\|(S x, x) .
\end{aligned}
$$

To conclude this paper we remark first that the significance of the Löwner-Heinz inequality is that it does not generally hold if $\alpha>1$. Second, Halmos's sharpening of Reid's inequality [4, p. 51, 244] was extended with some applications by the author in 6].

\section{REFERENCES}

[1] T. Furuta, A simplified proof of Heinz inequality and scrutiny of its equality, Proc. Amer. Math. Soc., 97(1986), 751-753. MR 87h:47016

[2] T. Furuta. $A \geq B \geq O$ assures $\left(B^{r} A^{p} B^{r}\right)^{1 / q} \geq B^{(p+2 r) / q}$ for $r \geq 0, p \geq 0, q \geq 1$ with $(1+2 r) q \geq p+2 r$, Proc. Amer. Math. Soc., 101(1987), 85-88. MR 89b:47028

[3] T. Furuta, Equivalence relations among Reid, Löwner-Heinz and Heinz-Kato inequalities, and extensions of these inequalities, Integr. Equ. Oper. Theory, 29(1997), 1-9. MR 98f:47022

[4] P. R. Halmos, Hilbert Space Problem Book, Van Nostrand, Princeton, N. J. 1967. MR 34:8178

[5] C.-S. Lin, On Heinz-Kato type characterizations of Furuta inequality, Nihonkai Math. J., 9(1998), 187-191. MR 99j:47021

[6] C.-S. Lin, On Halmos' sharpening of Reid's inequality, C. R. Math. Rep. Acad. Sci. Canada, 20(1998), 62-64. MR 99a:47015

[7] K. Löwner, Über monotone Matrixfunktionen, Math. Z., 38(1934), 177-216.

[8] W. T. Reid, Symmetrizable completely continuous linear transformations in Hilbert space, Duke Math. J., 18(1951), 41-56. MR 13:564b

Department of Mathematics, Bishop's University, Lennoxville, Quebec, Canada J1M $1 \mathrm{Z7}$

E-mail address: plin@ubishops.ca 